\title{
ASSOCIATED CONDITIONS AND SPECIFIC PREDICTS OF OBSTETRIC ANTIFOSPHOLIPID SYNDROME
}

\author{
RODRIGUES, Anielly Neri; ANDRADE, Izabela Cristina dos Santos²; LEON, Carlos Márcio \\ Moura Ponce de ${ }^{3}$
}

${ }^{1,2}$ Universidade Federal de Campina Grande, Centro de educação e saúde, Departamento de Farmácia, Sítio olho D'água, CEP 58175-000, Cuité - PB

(fone: (83) 3372-1900)

*Anielly Neri Rodrigues

e-mail: anielly_ne@hotmail.com

Received 26 October 2018; received in revised form 06 December 2018; accepted 10 December 2018

\section{RESUMO}

A síndrome antifosfolípide (SAF), ou doença de Hughes, uma doença sistêmica autoimune, é a mais comum das trombofilias adquiridas no adulto jovem. Na obstetrícia, ela é caracterizada clinicamente podendo estar associada a outras doenças. O objetivo desse trabalho foi identificar os preditores laboratoriais específicos de alto risco para perda fetal, tal como, as principais sintomatologias associadas. Esse estudo foi realizado através da análise de dados de 1557 pacientes grávidas diagnosticadas com síndrome antifosfolípide clássica. A condição mais associada às pacientes com SAF obstétrica no presente estudo foi à presença de aborto prévio, seguida de parto precoce, trombose, morte fetal, aborto recorrente e pré-eclâmpsia. Os preditores laboratoriais associados à síndrome antifosfolípide obstétrica foram o anticoagulante lúpico e a anticardiolipina $\mathrm{IgG} / \mathrm{lgM}$. É necessário que se faça um estudo para complementar o perfil das pacientes dessa síndrome na gravidez, pois, o desenvolvimento de anticorpos antifosfolípideos parecem ser frequentes em pacientes gravemente enfermos, sendo, porém necessários mais estudos para esclarecer seu papel patogênico e suas implicações na prática clínica.

Palavras chave: Síndrome Antifosfolipídica, trombofilia, Aspirantes a Aborto, Doenças Urogenitais Femininas e Complicações na Gravidez.

\begin{abstract}
The antiphospholipid syndrome (APS), or Hughes's disease, an autoimmune systemic disease, is the most common thrombophilias acquired in the young adult. In obstetrics, it is characterized clinically and may be associated with other diseases. The objective of this study was to identify the specific laboratory predictors of high risk for fetal loss, as well as the main associated symptomatologies. This study was performed through data analysis of 1557 pregnant patients diagnosed with classic antiphospholipid syndrome. The condition most associated with patients with obstetric SAF in the present study was the presence of previous abortion, followed by early delivery, thrombosis, fetal death, recurrent abortion and pre-eclampsia. The laboratory predictors associated with obstetric antiphospholipid syndrome were lupus anticoagulant and anticardiolipin $\lg \mathrm{G} / \mathrm{IgM}$. It is necessary to carry out a study to complement the profile of the patients of this syndrome in pregnancy, because the development of antiphospholipid antibodies appear to be frequent in critically ill patients, but more studies are necessary to clarify their pathogenic role and its implications in clinical practice.
\end{abstract}

Keywords: Antiphospholipid Syndrome, thrombophilia, Abortion Aspirants, Female Urogenital Diseases and Pregnancy Complications. 


\section{INTRODUÇÃO}

A síndrome antifosfolípide (SAF), ou doença de Hughes, é uma doença sistêmica autoimune, sendo a trombofilia adquirida mais comum em adultos jovens. Na obstétricia, ela é caracterizada clinicamente, pela ocorrência de anormalidades na gestação, podendo estar associada, à trombose venosa, arterial ou micro circulatório recorrente, principalmente em pacientes não tratadas (George; Erkan, 2009; Levy, 2011; Emmi et al., 2014; Cabrera et al., 2017; Santos et al., 2018).

A parte obstétrica da síndrome antifosfolípide pode resultar em vários efeitos adversos de gravidez, entre eles, morte fetal, aborto recorrente, parto prematuro, (principalmente em pacientes com pré-eclâmpsia grave ou insuficiência placentária), além de trombose em grávidas levando a uma taxa mais elevada de morbidade materna e fetal principalmente em pacientes com anticorpos antifosfolípides onde verifica-se um aumento do risco de aborto em pacientes grávidas. (Aliani et al., 2009; Bertolaccini et al., 2014; Abrahams; Chamley; Salmon, 2017). Segundo Jameil (2015), são a razão mais importante para o aborto recorrente, sendo essa a primeira complicação obstétrica relacionada a anticorpos antifosfolípides, causando de 5 a $10 \%$ dos abortos de repetição.

Como os antifosfolípides são um grupo heterogêneo de anticorpos, que possuem diversos mecanismos de ação, é pouco provável que a morbidade obstétrica seja causada por um único mecanismo, como o infarto e / ou a trombose (Damige et al., 2016).

Fosfolipídios estão presentes nas membranas celulares e são estruturalmente componentes lipídicos polares, os anticorpos antifosfolípides constituem um grupo heterogêneo de anticorpos produzidos por auto autoimunes. Pacientes com síndrome antifosfolipíde possuem anticorpos que reconhecem proteínas do plasma quando associadas à fosfolípidios de membrana. Os principais anticorpos antifosfolipídes são o anticoagulante lúpico, a anticardiolipina e a Bglicoproteínal (Miyakis et al., 2006; Soligo, 2007; Tincani et al, 2009; Branch; Gibson; Laat; Groot, 2010; Silver, 2010; Carvalho, 2018; Henry, 2012; Rand; Wolgast, 2012).

$$
\text { Uma superexpressão local do }
$$

autoantígeno alvo ( $\beta 2 \mathrm{GPI}$ ) para antifosfolipídios patogênicos foi sugerido para explicar pelo menos parte da patogênese das manifestações obstétricas. Os anticorpos antifosfolipídios ativam células endoteliais e plaquetas através da $32 \mathrm{GPI}$ (Groot; Derksen, 2005; Meroni et al., 2011; Abrahams; Chamley; Salmon, 2017).

Em todas as mulheres com anticorpos antifosfolípides positivos, é altamente contra indicado o uso de anticoncepcionais combinados, ou terapia de reposição hormonal, tromboses arteriais ou venosas serão tratadas com anticoagulante, como a varfarina ou heparinas não fracionada e de baixo peso molecular, o tratamento é utilizado por toda a vida e o alvo terapêutico é determinado pelo INR. Um grande desafio em relação à obstetrícia, é que, o tratamento de eventos obstétricos relacionados à antifosfolipídios não é apoiado por resultados consistentes de estudos bem planejados (Bertolaccini et al., 2014; Merashli et al., 2015).

O objetivo desse trabalho foi realizar uma revisão da literatura sobre a síndrome antifosfolipídica obstétrica, verificar as condições associadas a essa doença e identificar os preditores específicos de alto risco.

\section{DESENVOLVIMENTO}

\subsection{Metodologia aplicada}

O presente estudo tratou-se de uma revisão bibliográfica do tipo integrativa, o tipo de estudo foi a análise de uma série de casos. Foram utilizados artigos entre 2005 e 2018, na língua espanhola, inglesa e portuguesa. Foram confeccionados gráficos através de dados obtidos em artigos selecionados através do software Microsoft Excel (planilha de cálculo e elaboração de gráficos) Os descritores utilizados foram: síndrome antifosfolípide na gravidez, trombofilia adquirida, aborto de repetição, anticorpos antifosfolípides, evidenciando o anticorpo anticardiolipina, antiß2GP1 e anticoagulante lúpico.

\subsection{População de estudo}

Através da análise de dados dos artigos analisados, foram obtidas 1557 pacientes grávidas diagnósticas com síndrome antifosfolípide clássica.

\subsection{Fontes de informação}


Os bancos de dados utilizados foram periódicos como Scielo (Centro América Latina e Caribe em Ciências da Saúde), Pubmed, Bvs (biblioteca virtual em saúde), Saúde baseada em evidência, Portal do capes, comitês internacionais e nacionais de saúde, livros e dissertações.

\section{RESULTADOS E DISCUSSÃO:}

A condição mais associada às pacientes com SAF obstétrica foi histórico de aborto $(42,1 \%)$ seguido de parto precoce $(35,9 \%)$, trombose $(31,1 \%)$, morte fetal $(25,44 \%)$, aborto recorrente $(25,3 \%)$, pré-eclâmpsia $(21,73 \%)$, insuficiência placentária $(13,56 \%)$ e hipertensão $(11,0 \%)$ (Figura 1).

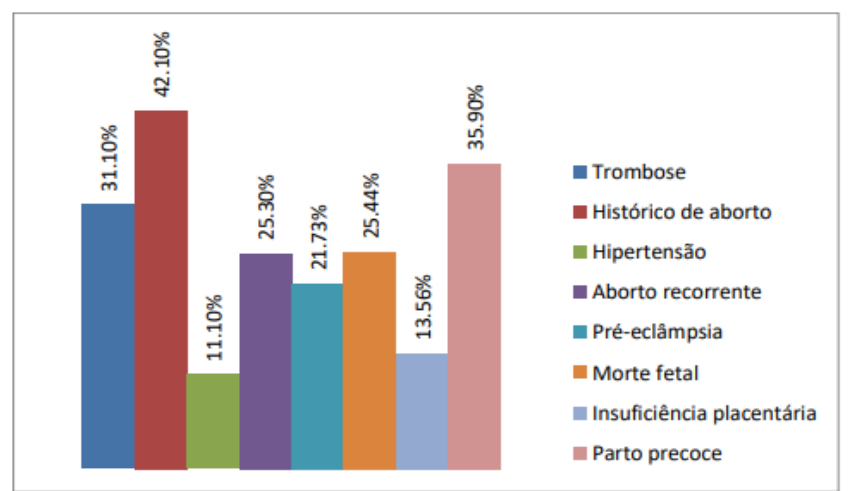

Figura 1. Condições associadas à SAF obstétrica

Em relação à SAF obstétrica concomitante com outras enfermidades a trombose esteve presente em $68 \%$ das pacientes estudadas em Mekinian et al. (2017) e em Lockschin et al.(2012), 53,57\% . Em Saccone et al. (2017) manifestou se $22,5 \%$ das pacientes. Já em Matsuk et al.(2014) e Latino et al.(2017) depararam se com valores, respectivamente de $7 \%$ e $4,7 \%$. A média geral, de acordo com o apresentado na figura 1 , foi de $31,1 \%$ indicando que há relação entre a SAF gestacional e a trombose, apenas Matsuk et al.(2014) e Latino et al.(2017) não confirmaram essa teoria.

A hipertensão ocorreu em $21,4 \%$ dos casos em Lockschin et al.(2012), já em Matsuk et al.(2014) ocorreu em 13,3\% das pacientes. Em Soh et al.(2013) apresentou se em 9,6\%, Mekinian et al. (2017) em 8\% e em Bouvier et al. (2013) foi encontrado 3,2\%, a média geral foi de $11,10 \%$ não indicando alta relação mostrando pouca associação entre essas doenças (Figura 1).
Com relação às manifestações características da parte obstétrica da SAF obstétrica, pré-eclâmpsia foi identificada principalmente no estudo de Saccone et al. (2017) em 68\% das pacientes com a síndrome antifosfolípide obstétrica. Houve uma queda significante para o seguinte que foi Lockschin et al.(2012) com 21,42\%, e um declínio ainda maior em Latino et al.(2017) 16\%, Mekinian et al. (2017) 10\%, Soh et al.(2013) 9,6\%, e Bouvier et al.( 2013) com 5,4\% (gráfico 1). A média geral desse evento no atual ensaio foi de $21,73 \%$ (Figura 1).

Abortos anteriores foram encontrados em $75 \%$ dos casos de Lockschin et al.(2012), esteve presente em $71 \%$ Mekinian et al. (2017). Em Saccone et al. (2017) manifestou se em 66,15\% e em 56,2\% de Soh et al.(2013). Latino et al. (2017) 31\% das pacientes sofreram abortos prévios, entretanto em Bowman et al.(2015) foram identificadas em $21 \%$, em Bouvier et al. (2013) apresentou se em 9,5\% . Apenas em 7\% das pacientes de Matsuk et al.(2014) esse episódio ocorreu. A média de todos os estudos foi de $42 \%$ sendo superior a encontrada na literatura (Santos, 2014).

Parto prematuro segundo Lockschin et al.(2012) esteve ligado a $57,14 \%$ dos casos, já Latino et al.(2017) foi encontrada $42,4 \%$ das enfermas e em Matsuk et al.(2014) ocorreu em $40 \%$. No entanto a partir de houve redução nesses valores $35 \mathrm{com}$ Mekinian et al. (2017) apresentando 22,5\%, Soh et al.(2013) 17,8\%. A média entre todos os autores foi de $35,9 \%$ (Figura 1).

Insuficiência placentária (Gráfico 1) Saconne et al. (2017) associou a 20,8\% dos casos, em Lockschin et al.(2012) estive presente em 14,28\%, Matsuk et al.(2014) em 13,3\% dos casos e em Bouvier et al. (2013) em 12,5\%, no entanto em Soh et al.(2013), havendo retraimento entre os valores anteriores estado presente em $6,8 \%$, a média encontrada nesse estudo foi de $13,56 \%$.

Morte fetal ocorreu em $50 \%$ das pacientes estudadas por Lockschin et al.(2012) e em proporção semelhante em Latino et al.(2017) com 46,2\%. Saconne et al.(2017) em $26 \%$, e em Bowman et al.(2015) mostrou taxa de 15,7\%, sendo similar a Soh et al.(2013) apresentando 13,6\%, em Bouvier et al. (2013) a taxa foi insignificante aparecendo em $1,2 \%$ apenas. A média de todas as gestantes estudadas foi de $25,44 \%$ (Figura 1). 
Entre os estudos analisados a taxa de abortos recorrentes corresponde a Lockschin et al.(2012) com 53,57\%. Já em Latino et al.(2017) foi 31\%, Mekinian et al. (2017) 30\%, Soh et al.(2013) 26\%, estes apresentaram valores semelhantes. Bowman et al.(2015) com 21\% houve pouco decaimento, entretanto em Bouvier et al., (2013) houve redução significativa sendo encontrada em $9,5 \%$. A média encontrada foi de 25\%, Salmon, Girardi e Lockshin (2007) afirmaram que aproximadamente um quarto das mulheres que sofrem abortos recorrentes tem $\mathrm{SAF}$, esse número levando em consideração que abortos recorrentes estiveram presentes em $25 \%$ das pacientes estudadas (Salmon; Girardi; Lockshin, 2007). Uma limitação desse estudo é que as características clínicas e a hipertensão não puderam ser estudadas separadamente, pois os autores não especificam quantas pacientes possuem mais de um sintoma (aborto prévio, parto precoce, trombose, morte fetal esteve presente em aborto recorrente, préeclâmpsia, insuficiência placentária), assim, as pacientes da síndrome antifosfolípide podem possuir mais de uma queixa associada ao quadro clínico, não sendo possível identificar a predominância destas doenças isoladamente.

Segundo análise de dados da figura 2, o anticorpo contra fosfolipídio mais encontrado foi a anticardiolipina $\operatorname{lgG} / \operatorname{lgM}(52,2 \%)$, em segundo lugar foi encontrado 0 anticoagulante lúpico (51\%), seguido da anticardiolipina IgM (45,6\%). Tanto o anticorpo anticardiolipina IgG, como o $\operatorname{lgM}(\mathrm{aCL})$ foram claramente relacionados às perdas fetais recorrentes antes de 24 semanas de gestação (Ruiz-irastorza; Khamashta, 2007).

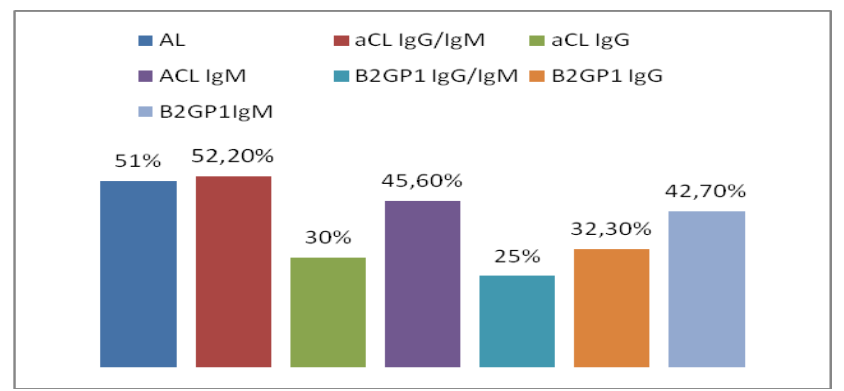

Figura 2. Distribuição de anticorpos

O nível de anticorpos anticardiolipina IgM foi significativamente maior no grupo préeclâmptico, com um nível de significância de $95 \%$, indicando anticorpos anticardiolipina na patogênese da pré-eclâmpsia como provável marcador sorológico inicial. Foi relatada uma associação entre aCL $\operatorname{lgG}$ (incluindo de baixa a moderada) em 153 abortos recorrentes ocorrendo com menos de 13 semanas de idade gestacional (Opatrny,2006; Pereira et al., 2015).

O anticoagulante lúpico esteve presente em $51 \%$ dos casos sendo apoiado por Lockschin et al.(2012), Soh et al., (2013), Latino et al.,(2017), Mekinian et al., (2017), Bouvier et al., 2013) porém esse valor pode ter sofrido influência em Bowman et al., (2015) onde não foi encontrado nenhum anticorpo anticoagulante, pode ter se dado, pois, a utilização de anticoagulante pelas pacientes, pode ter afetado a detecção desse antifosfolipídio, Saccone et al., (2017) também detectou baixos valores de anticoagulante lúpico. Ele foi definido como o principal preditor de resultado adverso da gravidez após 12 semanas de gestação em gravidezes associadas a antifosfolipídios, sua presença associada a anticorpos anticardiolipina $(\mathrm{aCL})$ e antiß2 glicoproteína I (antiß2GPI) está fortemente associada à trombose e à morbidade da gravidez, como geralmente é encontrado associado a outros antifosfolipídios o significado clínico do $\mathrm{AL}$ isolado é um achado incomum e controverso (Lockshin et al., 2012, Hoxha et al., 2017).

O B2GP1 lgM também esteve ligado $42,7 \%$ mostrando alta associação com resultados adversos de gravidez podendo ser explicada pela alta afinidade entre ela e a anticardiolipina (SOLIGO, 2017).

Os menos encontrados foram a $\beta 2 \mathrm{GP} 1$ lgG isolada e $\beta 2 G P 1 \lg G / \operatorname{lgM}$ e anticardiolipina IgG (figura 3).

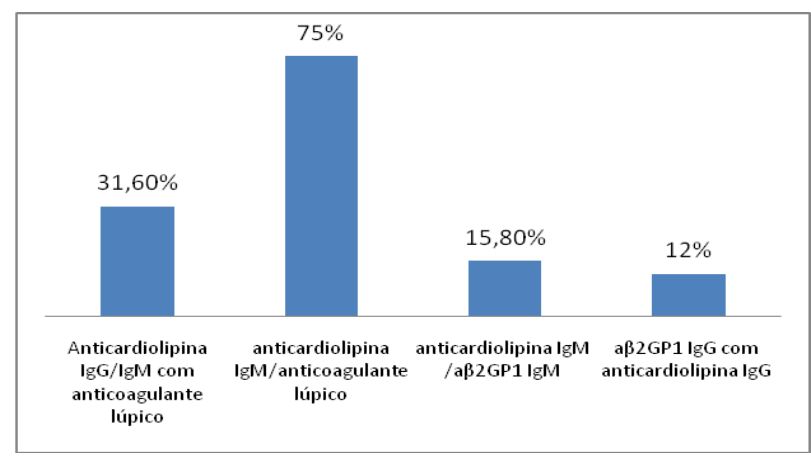

anticorpos

Figura 3. Associação de mais de

Anticardiolipina $\operatorname{lgG} / \lg M$ associada a anticoagulante lúpico foi relacionada a média de $31,6 \%$, a associação entre anticardiolipina $\operatorname{lgM}$ e $\mathrm{AL}$, anticardiolipina IgM e $\beta 2 \mathrm{GP} 1 \mathrm{IgM}$ e $\beta 2 \mathrm{GP} 1$ IgG/anticardiolipina IgG foram encontrados em 
apenas um estudo apresentando valores de $75 \%, 15,8 \%$ e $12 \%$ respectivamente, assim indica se mais estudos sobre a associação entre articardiolipina IgM e $\beta 2$ GP1(figura 3), indicando alta relevância no diagnóstico.

Mulheres com síndrome antifosfolipidica têm probabilidade aumentada de sofrer complicações quando há mais de um anticorpo antifosfolípide presente, apesar da utilização da terapia indicada com aspirina em baixas doses e heparina profilática de baixo peso molecular, a chance de um uma mulher triplo positivo dar à luz a um bebê nascido vivo é de apenas $30 \%$ para mulheres triplas positivas (Saccone et al., 2017).

\section{CONCLUSÕES:}

As pacientes avaliadas pelos artigos nesta revisão relataram queixas de aborto recorrente durante a anamnese. Foi identificada uma forte relação entre histórico prévio de aborto, parto precoce, um quarto das pacientes apresentou aborto espontâneo recorrente. A condição mais associada, às pacientes com SAF obstétrica, no presente estudo foi à presença de aborto prévio $(42,1 \%)$. Os preditores laboratoriais mais associados à síndrome antifosfolípide obstétrica foram, o anticoagulante lúpico e a anticardiolipina $\lg \mathrm{G} / \lg \mathrm{M}$, e a associação mais encontrada foi a de anticardiolipina IgM com AL, porém esse esteve presente em apenas um estudo. Sendo uma limitação desse estudo a falta de dados para associá-los.

\section{REFERÊNCIAS:}

1. Abrahams, Vikki M.; Chamley, Lawrence W.; Salmon, Jane E. Emerging Treatment Models in Rheumatology: Antiphospholipid Syndrome and Pregnancy. Arthritis \& Rheumatology, [s.l.], v. 69, n. 9, p.1710-1721, 18 jul. 2017.

2. Aliani, Natália Arruda et al. Síndrome do anticorpo antifosfolípide: relato de caso e revisão da literatura: Antiphospholipid antibody syndrome: case report and review of the literature. Rev. Méd. Minas Gerais, Minas Gerais, p.69-74, 2009.

3. Bertolaccini, Maria Laura et al. 14th International Congress on Antiphospholipid Antibodies Task Force.
Report on antiphospholipid syndrome laboratory diagnostics and trends.Autoimmunity Reviews, [s.I.], v. 13, n. 9, p.917-930, set. 2014.

4. Bouvier, s. Et al. Comparative incidence of pregnancy outcomes in treated obstetric antiphospholipid syndrome: the noh-aps observational study. Blood, [s.I.], v. 123, n. 3, p.404-413, 7 nov. 2013. American society of hematology.

5. Branch, d. W.; gibson, m.; silver, $r$. M.recurrentmiscarriage. New englandjournalof medicine, [s.I.], v. 363, n. 18, p.1740-1747, 28 out. 2010.

6. Cabrera, d. M. Et al. Controversias del síndrome de anticuerposantifosfolipídicos en obstetricia. Reumatologíaclínica, [s.I.], v. 13, n. 1, p.30-36, jan. 2017.

7. Carvalho, J. F. de. AnticorposAntifosfolípides. Disponível em:

8. <https://www.reumatologia.org.br/pacient es/orientacoes-ao-paciente/anticorposantifosfolipides/>. Acesso em: 15 jan. 2018.

9. Emmi, G. et al. An approach to differential diagnosis of antiphospholipid antibody syndrome and related conditions. The scientific world journal, v. 2014, p.1-8, 2014. Hindawi limited.

10. George, d.; erkan, d. Antiphospholipid syndrome. Progress in cardiovascular diseases, v. 52, n. 2, p.115-125, set. 2009. Elsevier bv.

11. Groot, P. G.; Derksen, R. H. W. M.. Pathophysiology of the antiphospholipid syndrome. Journal Of Thrombosis And Haemostasis, [s.I.], v. 3, n. 8, p.18541860, ago. 2005.

12. Henry, J. B.; Abordagem laboratorial do riscotrombó tico. In: HENRY, John Bernar. Diagnósticos Clínicos e Tratamento por Métodos Laboratoriais de Henry. 21. ed. S.I: Manole, 2012.

13. Jameil, n. Et al. Incidence of anticardiolipin antibodies and lupus anticoagulant factor among women experiencing unexplained recurrent abortion and intrauterine fetal death. International journal of clinical and experimental pathology, arábiasaudita, v. 8, n. 3, p.3204-3209, mar. 2015. 
14. Latino, j o et al. Pregnancy failure in patients with obstetric antiphospholipid syndrome with conventional treatment: the influence of a triple positive antibody profile. Lupus, [s.I.], v. 26, n. 9, p.983-988, 7 fev. 2017. Sage publications.

15. Levy, r.; jesús, g.; jesús, nr. Obstetric antiphospholipid syndrome: still a challenge. Lupus, v. 19, n. 4, p.457-459, 30 mar. 2010.

16. Lockshin, m. D. Et al. Prediction of adverse pregnancy outcome by the presence of lupus anticoagulant, but not anticardiolipin antibody, in patients with antiphospholipid antibodies. Arthritis \& rheumatism, [s.I.], v. 64, n. 7, p.23112318, 26 jun. 2012. Wiley.

17. Matsuki, yuko et al. Clinical features and pregnancy outcome in antiphospholipid syndrome patients with history of severe pregnancy complications. Modern rheumatology, [s.I.], v. 25, n. 2, p.215-218, 22 ago. 2014. Informa uk limited.

18. MERASHLI, Mira et al. Antiphospholipid syndrome: an update. European Journal Of Clinical Investigation, [s.I.], v. 45, n. 6 , p.653-662, 24 abr. 2015.

19. Mekinian, a. Et al. Refractory obstetrical antiphospholipid syndrome: features, treatment and outcome in a european multicenter retrospective study. Autoimmunity reviews, [s.I.], v. 16, n. 7 , p.730-734, jul. 2017.

20. MERONI, Pier Luigi et al. Pathogenesis of antiphospholipid syndrome: understanding the antibodies. Nature Reviews Rheumatology, [s.I.], v. 7, n. 6, p.330-339, 10 maio 2011.

21. Miyakis, S. etal.International consensus statement on an update of the classification criteria for definite antiphospholipid syndrome (APS). Journal Of Thrombosis And Haemostasis, v. 4, n. 2, p.295-306, fev. 2006.

22. Laat, B. de; Groot, P.G. de.; Autoantibodies Directed Against Domain I of Beta2- Glycoprotein I. Current Rheumatology Reports, v. 13, n. 1, p.7076, 3 nov. 2010

23. Opatrny, I. Association between antiphospholipid antibodies and recurrent fetal loss in women without autoimmune. The journal of rheumatology, [s.I], v. 33, n. 11, p.214-221, set. 2006., opatrny. Association between antiphospholipid antibodies and recurrent fetal loss in women without autoimmune. The journal of rheumatology, [s.I], v. 33, n. 11, p.214221, set. 2006.

24. Pereira, k. N. Et al. Level of reactivity of igm anticardiolipin antibodies in preeclampsia: a likely early serologic marker. Jornal brasileiro de patologia e medicina laboratorial, [s.I.], v. 51, n. 1 , p.287-297, 2015. Gn1 genesis network.

25. Rand J.H.; Wolgast L.R. Dos and don'ts in diagnosing antiphospholip- id syndrome. Hematology Am Soc Hematol Educ Program. P. 455-459. 2012.

26. Saccone, g. Etal.antiphospholipid antibody profile based obstetric outcomes of primary antiphospholipid syndrome: the pregnants study. American journal of obstetrics and gynecology, v. 216, n. 5, p.1-42, maio 2017.

27. Salmon, jane e; girardi, guillermina; lockshin, michael $d$. The antiphospholipid syndrome as a disorder initiated by inflammation: implications for the therapy of pregnant patients. Nature clinical practice rheumatology, [s.I.], v. 3, n. 3, p.140-147, mar. 2007. Springer nature.

28. Santos, f. C. Et al. Anticoagulação na gravidez. Revista hospital universitário pedro ernesto, v. 14, n. 2, dez. 2015. Issn 1983-2567. Acesso em: 01 abr. 2018.

29. Soh, m. C. Et al. Persistent antiphospholipid antibodies do not contribute to adverse pregnancy outcomes. Rheumatology, [s.I.], v. 52, n. 9, p.1642-1647, 16 maio 2013. Oxford university press (oup).

30. Soligo, Adriana de Góes e Silva et al. Prevalência dos fatores trombofílicos em mulheres com infertilidade. Revista Brasileira de Ginecologia e Obstetrícia, [s.l.], v. 29, n. 5, p.235-240, maio 2007.

31. Tincani, a. Et al. Neonatal effects of maternal antiphospholipid syndrome. Current rheumatology reports, [s.I.], v. 11,
1 ,
p.70-76
fev.
2009. 
PERIÓDICO TCHÊ QUÍMICA • www.periodico.tchequimica.com • Vol. 16 N. 31.

- ISSN 1806-0374 (impresso) • ISSN 1806-9827 (CD-ROM) • ISSN 2179-0302 (meio eletrônico) (C) 2019. Porto Alegre, RS. Brasil

The Periódico Tchê Química (ISSN: 1806-0374; 2179-0302) is an open-access journal since 2004. Journal DOI: 10.52571/PTQ. http://www.tchequimica.com. This text was introduced in this file in 2021 for compliance reasons.

() The Author(s)

OPEN ACCESS. This article is licensed under a Creative Commons Attribution 4.0 (CC BY 4.0) International License, which permits use, sharing, adaptation, distribution, and reproduction in any medium or format, as long as you give appropriate credit to the original author(s) and the source, provide a link to the Creative Commons license, and indicate if changes were made. The images or other third-party material in this article are included regulation or exceeds the permitted use, you will need to obtain permission directly from the copyright holder. To view a copy of this license, visit http $/ /$ creativecommons org/licenses/by/4. $0 /$ regulation or exceeds the permitted use, you will need to obtain permission directly from the copyright holder. To view a copy of this license, visit http://creativecommons.org/licenses/by/4.0/ 